\title{
Irisin Gene Polymorphism Rs3480 and Rs1746661and Obesity in Egyptian Populations
}

\author{
Azza M. Abdu Allah ${ }^{1}$, Eman Masoud Abd El Gayed ${ }^{* 1}$, Sahar AF. Hammoudah ${ }^{2}$, \\ Lama Mohamed El-Attar ${ }^{3}$, Walid A. Shehab-Eldin ${ }^{4}$ \\ ${ }^{I}$ Medical Biochemistry Department, Faculty of Medicine, Menoufia University, Egypt \\ ${ }^{1 *}$ Medical Biochemistry Department, Faculty of Medicine, Menoufia University, Egypt \\ ${ }^{2}$ Clinical Pathology Department, Faculty of Medicine, Tanta University, Egypt. \\ ${ }^{3}$ Human Genetics Department, Medical Research Institute, Alexandria University, Egypt \\ ${ }^{4}$ Internal Medicine Department, Faculty of Medicine, Menoufia University, Egypt.
}

\begin{abstract}
The prevalence of obesity is rising worldwide, and presenting a public health problem. Irisin is a novel myokine that is, proteolytically processed from the product of the FNDC5 gene. This study aims at finding out the distribution of FNDC5 rs3480 (A/G) and rs1746661 (G/T) polymorphisms in obese individuals and their association with other clinical and laboratory variables in these persons. This study was done on 141 subjects divided into 2 groups: group I obese group (71) and group II; control group (70). All subjects were submitted to full history taking, general clinical examination and laboratory investigations including BMI, lipid profile, fasting blood glucose, glycated hemoglobin (HbAlc) and genotyping of rs3480 (A/G) and rs1746661 (G/T) gene polymorphisms. There was a significant statistical difference between the two groups regarding genotype frequency of FNDC5 (Irisin precursor) $r s 3480(\mathrm{~A} / G)$ and $r s 174661(\mathrm{G} / \mathrm{T})$. Regarding $r s 3480(\mathrm{~A} / \mathrm{G})$, there was a high frequency of AA genotype and A allele among the control group, while GG genotype and $G$ allele had the highest frequency among obese group. Regarding $r s 1746661$ (G/T), there was a high frequency of GG genotype and $G$ allele among the control group, while TT genotype and $T$ allele had the highest frequency among obese group. Multivariate logistic regression for risk of obesity showed that the most common risk factor is BMI OR; 413.9 (6.7-492.6), followed by HBAIC OR; 67.1 (0.72-256.8), Tc OR; 53.6 (3.8-44.5), LDL-C OR; 26.8 (1.0395.1).

Conclusion: Our results indicate that $G G$ genotype and $G$ allele of $r s 3480(A / G)$ polymorphism and TT genotype and T allele of rs $1746661(G / T)$ might be genetic risk factor for obesity.
\end{abstract}

Keywords: Obesity, Irisin, gene polymorphism, allele, FNDC5

\section{Introduction}

Obesity is a complex disorder with strong genetic components and has an impact on morbidity and mortality. Studies have suggested a genetic cause to variation in body mass index (BMI) (1).Various health risks are related with increasing BMI. An individual's predisposition to obesity is determined by interactions between genetic and environmental factors (2). Insulin resistance is commonly associated with obesity (3). Obesity is considered as independent risk factor for many serious diseases such as metabolic, cardiovascular, respiratory and gastrointestinal disorders (4).

The co-occurrence of metabolic risk factors for both Type 2 Diabetes and CVD (abdominal obesity, hyperglycemia, dyslipidemia, and hypertension) suggested the existence of a "metabolic syndrome. The metabolic syndrome is a common metabolic disorder that results from the increasing prevalence of obesity. The pathophysiology seems to be largely attributable to insulin resistance with excessive flux of fatty acids implicated. A proinflammatory state probably contributes to the syndrome (5). Several adipocyte and hepatocyte derived factors, so called adipokines and hepatokines, have been suggested as a critical link between obesity, metabolic and cardiovascular disease. Nowadays, proteins which are secreted from myocytes that called myokines, have been introduced in the field of cardiovascular research (6).It has been suggested that irisin one of the myocyte-secreted proteins may be beneficial not only for the treatment of obesity and T2DM but also for a wide range of pathological conditions characterized by an imbalance between energy intake and expenditure (7). Irisin induces a 'browning' of white adipose tissue with marked elevated expression of uncoupling protein 1 and other brown fat genes(8). Polymorphisms in the gene encoding irisin, fibronectin type III domain containing 5 (FNDC5), is associated with Type 2 Diabetes Mellitus (T2DM) and related disorders (7). In this study we tried to clarify the distribution of FNDC5 rs3480 (A/G) and rs1746661 (G/T) Polymorphisms in obese subjects and their association with other clinical and laboratory variables. 


\section{SUBJECTS:}

\section{Subjects And Methods:}

This case-control study included (141) subjects; (71) obese patients and (70) healthy, age- and sexmatched subjects as a control group. Subjects were selected from the Internal Medicine Outpatient Clinic, Menoufia University and Tanta Hospital, Egypt. All subjects were subjected to complete history taking and physical examination including anthropometric measurements. Estimation of body mass index (BMI) $\mathrm{kg} / \mathrm{m}^{2}(9)$, total cholesterol(TC), triglycerides (TG), low density lipoprotein (LDL-c), high density lipoprotein (HDL-c), triglycerides (TG), fasting blood glucose, glycated hemoglobin (HbA1c) and genotyping of FNDC5 rs3480 (A/G) and rs1746661 (G/T) using the TaqMan allelic Discrimination assay technique (real time PCR).

\section{Sample collection and assay procedure:}

Prior to the collection of samples, written consent forms (approved by the Committee of Human Rights in Research at Menoufia University) were obtained from all studied subjects. Subjects with comorbidities that needed medical attention or with medical complications (diabetes mellitus, coronary artery disease, nephropathy, thyroid diseases and end stage renal or liver disease) were excluded from the study.

After 12 hours overnight fasting, $8 \mathrm{ml}$ of venous blood were withdrawn from every subject by sterile veinpuncture and divided into three tubes; $4 \mathrm{ml}$ of blood were transferred into two EDTA tubes: one of them was used for quantitative colorimetric determination of glycated hemoglobin using kits supplied by Teco diagnostics, USA(10), the other EDTA tube for genotyping of FNDC5gene, One ml of blood was transferred into sodium fluoride tube for enzymatic colorimetric determination of blood glucose. Blood glucose was determined by enzymatic colorimetric test (11). Three $\mathrm{ml}$ of blood was transferred into plain tubes from which we took serum after centrifugation. Sera were used for colorimetric determination of TC(12),HDL(13),LDL(14),TG(15)

DNA was extracted from whole blood using Zymo Research Quick-g DNA Mini PrepGenomic DNA purification kit,( USA).FNDC5gene was genotyping using the TaqMan allelic Discrimination assay technique that detects variants of single nucleic acid sequence. Using the maxima probe qPCR Master Mix (2X), primers and probes were purchased from Applied BioSystem:1) rs3480 5'AGCTCTTGTAGACCGGAAGGAA3' (Fprimer), 5'TGGTCCCCAAGCCAGAGA3' (R-primer), FAM 5'CCATCACCCAATGAC3', and VIC5'AGCCATCACCTAATGAC3'; 2) rs1746661 — 5'CTGCCCTGTCCAATGAGTGA3' (F-primer), 5' ACACTTCTCGATCTCTGGGTCTTT3' (R-primer), FAM-5' CCTTGCTCGAAGGAG3', and VIC5'CTTGCGCGAAGGAG3'. The genotyping reaction mix was prepared by mixing 10ul master mix, 1.25 of the genotyping assay mix (probe and primers) and $3.75 \mathrm{ul}$ of DNAase free water. For each unknown reaction, 5 $\mathrm{ul}(0.1 \mathrm{ug} / \mathrm{ul})$ of genomic DNA template and for negative control reaction $5 \mathrm{ul}$ of DNA free water was added.The cycling parameters were set as follows: initial denaturation step at $95^{\circ} \mathrm{C}$ for $10 \mathrm{~min}, 40$ cycles of denaturation at $94^{\circ} \mathrm{C}$ for $15 \mathrm{~s}$, annealing collection at $50^{\circ} \mathrm{C}$ for $60 \mathrm{~s}$ and extension at $72^{\circ} \mathrm{C}$ for $2 \mathrm{~min}$ and a final extension step at $72^{\circ} \mathrm{C}$ for min using 57500 Real Time PCR system (Applied BioSystem Co. Ltd, San Francisco Bay Area, USA).

\section{Statistical Analysis:}

Results were collected, tabulated and statistically analyzed by IBM personal computer and statistical package SPSS version 20 (IBM Corporation, Armonk, NY, USA). Chi-square test ( $\chi 2)$ was used to study the association between two qualitative variables. Odds ratio (OR) describes the probability .Student's $t$-test was used for comparison between two groups having quantitative variables. Analysis of variance (ANOVA) $(F)$ test was used for comparison between three or more groups having quantitative variables. Multiple regression analysis was performed to calculate the effects of risk factors as independent ORs with the effects of other confounders removed. $P$-value $<0.05$ was statistically significant.

\section{Results}

The study was conducted on a total number of 141 subjects divided into two groups as follows; 71 obese subjects as group I and 70 healthy persons as group II. There was a statistically significant difference between the two studied groups regarding serum FBG,HBA1C, TC,TG ,LDLc, HDLc and BMI $(P<0.001)$, while there was no significant difference as regarding age and sex (Table 1). .

As regards FNDC5 rs3480 (A/G)genotype distribution between the two studied groups; it showed a significant statistical difference, with increased frequency of the GG and AG genotypes and G allele in obese group and increased AA genotype and A allele frequency in the control group $(P<0.001$; Table 2 and Figure 1,2). In FNDC5 rs1746661 (G/T) genotype the distribution between the two studied groups showed a significant difference, with increased frequency of the TT and GT genotypes and T allele in obese group and increased GG genotype and $\mathrm{G}$ allele frequency in the control group $(P<0.001$; Table 2 and Figure 3,4). The results also showed that the GG genotype of FNDC5 rs3480 (A/G)increases the risk of obesity by 6.4fold and AG genotype increases the risk by 3.3 fold, while the $\mathrm{G}$ allele increases the risk by 2.7 fold, as shown in Table 
2.As regards TT genotype of FNDC5 rs1746661 (G/T) increases the risk of obesity by 7.9 fold and GT genotype increases the risk by 3.3 fold, while the $\mathrm{T}$ allele increases the risk by 4-fold, as shown in Table 2 . In group I, we compared the three different genotypes of FNDC5 rs1746661 (G/T) (GG, GT and TT) regarding serumFBG,HbA1 ${ }_{C}, T C, T G, L D L c$, HDLc and BMI. Subjects with TT genotype showed higher levels of TC, TG, LDL-c and BMI, while HDL-c was higher in subjects with GG genotype Table 3. In group I ; we compared the three different genotypes of FNDC5 rs3480 (AA, AG and GG) regarding serum FBG,HBA1, TC,TG ,LDLc, HDLc and BMI. Subjects with GG genotype showed higher levels of TC, TG, LDL-c and BMI, while HDL-c was higher in subjects with AA genotype, as shown in Table 4. Multivariate logistic regression for risk of obesity showed that the most common risk factor is BMI OR; 413.9 (6.7-492.6), followed by HBA1C OR; 67.1 (0.72-256.8), TC OR; 53.6 (3.8-44.5), LDL-C OR; 26.8 (1.03-95.1)(Table 6).

Table 1: Demographic and clinical characteristics of the studied groups.

\begin{tabular}{|l|l|l|l|l|}
\hline & $\begin{array}{l}\text { Obese } \\
\mathrm{N}=71\end{array}$ & $\begin{array}{l}\text { Control } \\
\mathrm{N}=70\end{array}$ & T test & P value \\
\hline Age (years) & $24.3 \pm 4$ & $24.8 \pm 3.03$ & 0.751 & 0.454 \\
\hline Sex male & $35(49.3 \%)$ & $40(57.1 \%)$ & $0.872^{*}$ & 0.35 \\
female & $36(50.7 \%)$ & $30(42.9 \%)$ & & \\
\hline BMI $\left(\mathrm{kg} / \mathrm{m}^{2}\right)$ & $37.3 \pm 6.5$ & $20.2 \pm 1.8$ & 21.1 & $<0.001$ \\
\hline FBS $(\mathrm{mg} / \mathrm{dl})$ & $90.5 \pm 9.1$ & $85.3 \pm 3.2$ & 4.5 & $<0.001$ \\
\hline HBA1 $\%$ & $5 \pm 0.5$ & $4.6 \pm 0.3$ & 6.4 & $<0.001$ \\
\hline Total Cholesterol $(\mathrm{mg} / \mathrm{dl})$ & $254.3 \pm 28.02$ & $156.8 \pm 10.4$ & 27.3 & $<0.001$ \\
\hline TG(mg/dl) & $158.9 \pm 21.2$ & $60.8 \pm 8.3$ & 35.9 & $<0.001$ \\
\hline LDL-C(mg/dl) & $196.1 \pm 28.4$ & $92 \pm 12.9$ & 28 & $<0.001$ \\
\hline HDL-C(mg/dl) & $26.6 \pm 2.2$ & $51.2 \pm 7.2$ & 27.4 & $<0.001$ \\
\hline
\end{tabular}

$* \times 2$

Table 2: Comparison of FNDC5genotypes between the studied Groups.

\begin{tabular}{|c|c|c|c|c|c|}
\hline & $\begin{array}{l}\text { Obese } \\
N=71\end{array}$ & $\begin{array}{l}\text { Control } \\
\mathrm{N}=70\end{array}$ & $\mathrm{X} 2$ & $\mathrm{P}$ value & $\mathrm{OR}(\mathrm{CI})$ \\
\hline $\begin{array}{l}\text { RS3480 } \\
\text { AA* } \\
\text { AG } \\
\text { GG }\end{array}$ & $\begin{array}{l}12(16.9 \%) \\
35(49.3 \%) \\
24(33.8 \%)\end{array}$ & $\begin{array}{l}32(45.7) \\
28(40 \%) \\
10(14.3 \%)\end{array}$ & 15.6 & $<0.001$ & $\begin{array}{l}3.3(1.4-7.6) \\
6.4(2.4-17.3)\end{array}$ \\
\hline $\begin{array}{l}\text { Allells } \\
A^{*} \\
\text { G }\end{array}$ & $\begin{array}{l}59(41.6 \%) \\
83(58.4 \%)\end{array}$ & $\begin{array}{l}92(65.7 \%) \\
48(34.3 \%)\end{array}$ & 16.5 & $<0.001$ & $2.7(1.7-4.4)$ \\
\hline $\begin{array}{l}\text { RS1746661 } \\
\text { TT } \\
\text { GT } \\
\text { GG* }\end{array}$ & $\begin{array}{l}37(52.1 \%) \\
22(31 \%) \\
12(16.9 \%)\end{array}$ & $\begin{array}{l}14(20 \%) \\
20(28.6 \%) \\
36(51.4 \%)\end{array}$ & 22.5 & $<0.001$ & $\begin{array}{l}7.9(3.2-19.4) \\
3.3(1.3-8)\end{array}$ \\
\hline $\begin{array}{l}\mathrm{G}^{*} \\
\mathrm{~T}\end{array}$ & $\begin{array}{l}46(32.4 \%) \\
96(67.6 \%)\end{array}$ & $\begin{array}{l}92(65.7 \%) \\
48(34.3 \%)\end{array}$ & 31.3 & $<0.001$ & $4(2.4-6.5)$ \\
\hline
\end{tabular}

\section{RS3480 polymorphism}

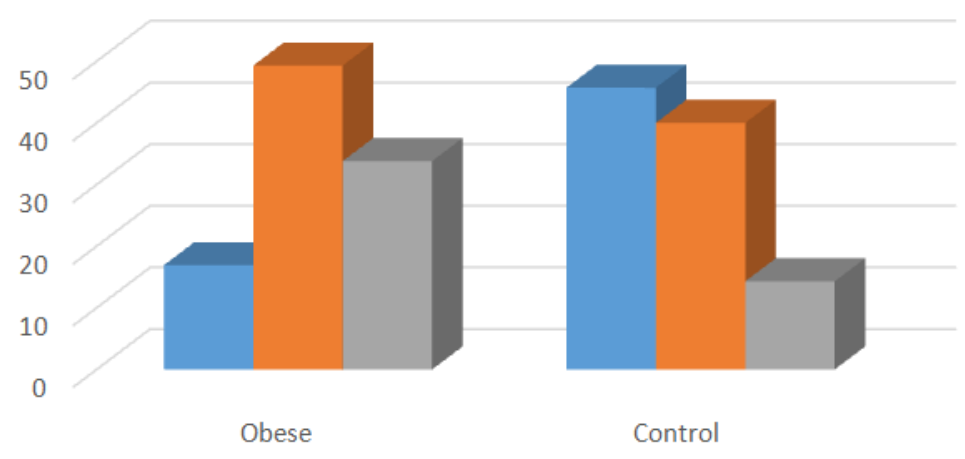

$A \mathrm{AA}=\mathrm{AG}=\mathrm{GG}$

Figure 1: Genotype distribution of the FNDC5 rs3480 (A/G) polymorphism between two studied groups. 


\section{RS3480 polymorphism allels}

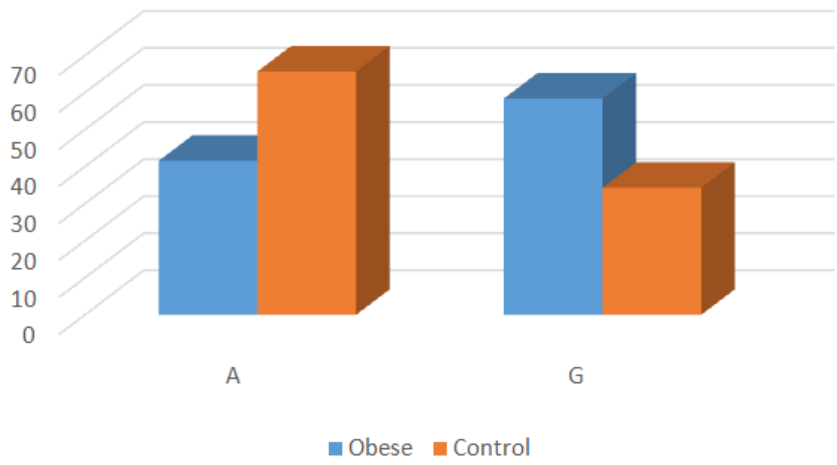

Figure 2: Allelic distribution of the FNDC5 rs3480 (A/G) polymorphism between two studied groups.

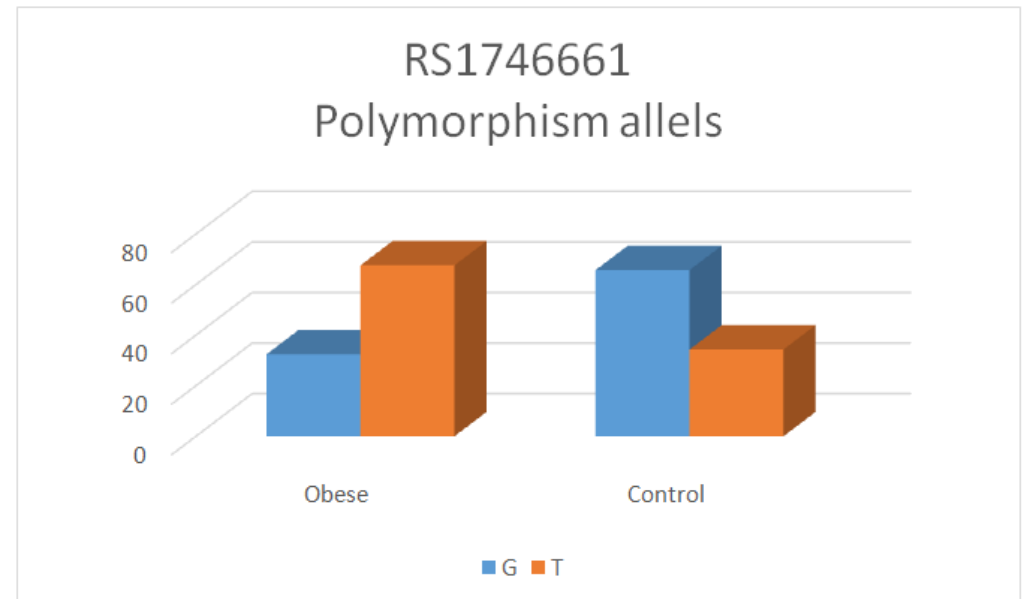

Figure 3: Allelic distribution of the FNDC5 rs1746661 (G/T) polymorphism between two studied groups.

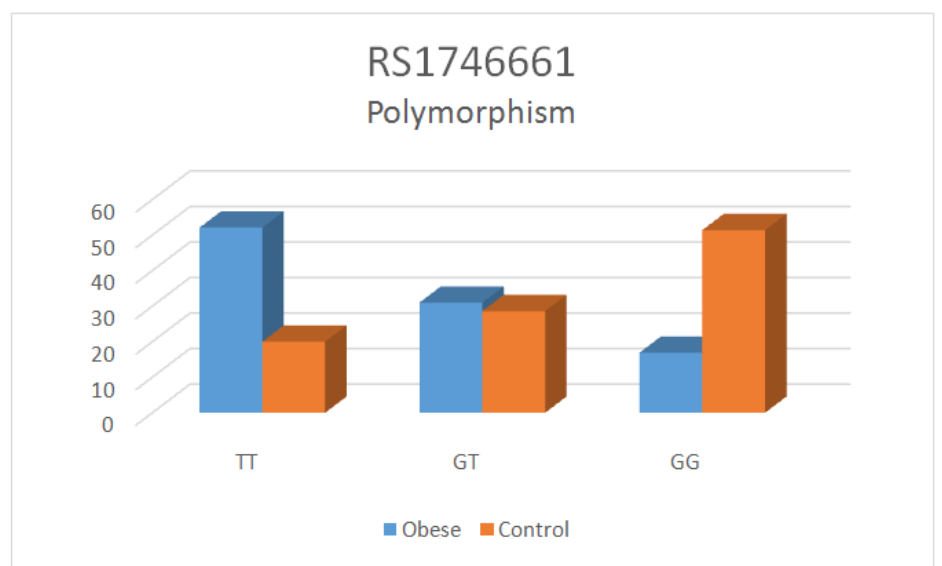

Figure 4: Genotype distribution of the FNDC5 rs1746661 (G/T) polymorphism between two studied groups.

Table 3: Biochemical parameters of the studied subjects with obesity in different genotypes of FNDC5 rs1746661 (G/T).

\begin{tabular}{|c|c|c|c|c|c|}
\hline & \multicolumn{3}{|c|}{ rs1746661 } & \multirow[t]{2}{*}{ F test } & \multirow[t]{2}{*}{$\mathrm{P}$ value } \\
\hline & TT & GT & GG & & \\
\hline $\operatorname{BMI}\left(\mathrm{kg} / \mathrm{m}^{2}\right)$ & $34.8 \pm 11.2$ & $26.9 \pm 7.9$ & $24.1 \pm 5.7$ & 20.5 & $<0.001$ \\
\hline $\mathrm{FBS}(\mathrm{mg} / \mathrm{dl})$ & $90.2 \pm 8.5$ & $88.3 \pm 6.8$ & $84.9 \pm 5.1$ & 7.3 & 0.001 \\
\hline $\mathrm{HBA} 1_{\mathrm{C}} \%$ & $4.9 \pm 0.5$ & $4.8 \pm 0.4$ & $4.7 \pm 0.4$ & 2.04 & 0.133 \\
\hline Total Cholesterol(mg/dl) & $238.7 \pm 54.9$ & $206.7 \pm 42.8$ & $170.3 \pm 34.5$ & 28.4 & $<0.001$ \\
\hline
\end{tabular}


Irisin Gene Polymorphism Rs3480 And Rs1746661and Obesity In Egyptian Populations

\begin{tabular}{|l|l|l|l|l|l|}
\hline TG(mg/dl) & $137.2 \pm 48.2$ & $111 \pm 47.5$ & $80.8 \pm 43.2$ & $18.3^{*}$ & $<0.001$ \\
\hline LDL-c(mg/dl) & $180.1 \pm 56.1$ & $144.4 \pm 47.4$ & $106.5 \pm 37.5$ & 29.3 \\
\hline HDL-C(mg/dl) & $30.8 \pm 9.7$ & $39.3 \pm 12.8$ & $46.9 \pm 12.6$ & $<0.001$ & 23.6 \\
\hline
\end{tabular}

*K test

Table 4: Biochemical parameters of the studied subjects with obesity in different genotypes of FNDC5 rs3480

(A/G).

\begin{tabular}{|c|c|c|c|c|c|}
\hline & \multicolumn{3}{|c|}{ rs3480 } & \multirow[t]{2}{*}{ F test } & \multirow[t]{2}{*}{$P$ value } \\
\hline & AA & $\mathrm{AG}$ & GG & & \\
\hline $\operatorname{BMI}\left(\mathrm{kg} / \mathrm{m}^{2}\right)$ & $26.1 \pm 10.7$ & $28.7 \pm 7.9$ & $32.4 \pm 10.8$ & $4.2 *$ & 0.017 \\
\hline FBS(mg/dl) & $86.9 \pm 5.7$ & $88.2 \pm 8.1$ & $88.4 \pm 7.5$ & 0.5 & 0.575 \\
\hline $\mathrm{HBA} 1_{\mathrm{C}} \%$ & $4.6 \pm 0.4$ & $4.8 \pm 0.4$ & $4.9 \pm 0.5$ & 3.7 & 0.026 \\
\hline Total Cholesterol (mg/dl) & $187.8 \pm 47$ & $207.4 \pm 52.4$ & $226.5 \pm 55.9$ & 5.4 & 0.005 \\
\hline TG(mg/dl) & $90.4 \pm 46.2$ & $112.4 \pm 51.1$ & $131.9 \pm 51.6$ & 6.8 & 0.002 \\
\hline LDL-c(mg/dl) & $126.3 \pm 48.5$ & $146.9 \pm 55.9$ & $163.3 \pm 62.3$ & $4.4^{*}$ & 0.014 \\
\hline HDL-c(mg/dl) & $42.2 \pm 11.3$ & $38.1 \pm 13.5$ & $35.7 \pm 15.2$ & $2.5^{*}$ & 0.09 \\
\hline
\end{tabular}

*K test

Table 5: Comparison of all studied biochemical and genetic parameters between obese and control group regarding sex

\begin{tabular}{|c|c|c|c|c|c|c|}
\hline & \multicolumn{3}{|l|}{ Obese } & \multicolumn{3}{|l|}{ Control } \\
\hline & Male(36) & Female (35) & $\mathrm{P}$ value & Male (30) & Female (40) & $\mathrm{P}$ value \\
\hline $\begin{array}{l}\text { RS3480 } \\
\text { AA } \\
\text { GA } \\
\text { GG }\end{array}$ & $\begin{array}{l}6(17.1) \\
18(51.4) \\
11(31.4)\end{array}$ & $\begin{array}{l}6(16.7) \\
17(47.2) \\
13(36.1) \\
\end{array}$ & 0.913 & $\begin{array}{l}15(50) \\
15(50) \\
0\end{array}$ & $\begin{array}{l}17(42.5) \\
13(32.5) \\
10(25) \\
\end{array}$ & 0.011 \\
\hline $\begin{array}{l}\text { RS1746661 } \\
\text { TT } \\
\text { GT } \\
\text { GG } \\
\end{array}$ & $\begin{array}{l}20(57.1) \\
10(28.6) \\
5(14.3) \\
\end{array}$ & $\begin{array}{l}17(47.2) \\
12(33.3) \\
7(19.4) \\
\end{array}$ & 0.689 & $\begin{array}{l}10(33.3) \\
0 \\
20(66.7)\end{array}$ & $\begin{array}{l}4(10) \\
20(50) \\
16(40) \\
\end{array}$ & $<0.001$ \\
\hline $\operatorname{BMI}\left(\mathrm{kg} / \mathrm{m}^{2}\right)$ & $37.3 \pm 6.9$ & $37.2 \pm 6.1$ & 0.989 & $21.5 \pm 0.8$ & $19.2 \pm 1.8$ & $<0.001$ \\
\hline FBS(mg/dl) & $87.7 \pm 9.6$ & $93.3 \pm 7.5$ & 0.008 & $83.3 \pm 3.8$ & $86.7 \pm 1.5$ & $<0.001$ \\
\hline HBA1C $\%$ & $4.9 \pm 0.5$ & $5.1 \pm 0.4$ & 0.037 & $4.7 \pm 0.3$ & $4.5 \pm 0.2$ & 0.002 \\
\hline Total Cholesterol (mg/dl) & $246.1 \pm 29.2$ & $262.7 \pm 24.4$ & 0.012 & $152.5 \pm 7.5$ & $160 \pm 11.2$ & 0.002 \\
\hline $\mathrm{TG}(\mathrm{mg} / \mathrm{dl})$ & $167.8 \pm 24.5$ & $149.7 \pm 11.9$ & $<0.001$ & $56.7 \pm 6.9$ & $64 \pm 7.9$ & $<0.001$ \\
\hline LDL-C(mg/dl) & $185.5 \pm 28.2$ & $207.1 \pm 24.4$ & 0.001 & $92.2 \pm 9.6$ & $91.9 \pm 14.9$ & 0.926 \\
\hline HDL-C(mg/dl) & $27.2 \pm 2.3$ & $25.9 \pm 2$ & 0.01 & $47.2 \pm 6.5$ & $54.2 \pm 6.3$ & $<0.001$ \\
\hline
\end{tabular}

Table 6: Multivariate logistic regression for risk of obesity.

\begin{tabular}{|l|l|l|l|l|}
\hline & B & P value & OR & CI(95\%) \\
\hline RS3480 & & & & \\
AA & & & & \\
GA & 0.62 & 0.726 & 0.537 & $0.017-17.4$ \\
GG & 2.8 & 0.07 & 15.9 & $0.815-312.1$ \\
\hline RS1746661 & & & & \\
TT & 1.9 & 0.239 & 7.1 & $0.273-183.3$ \\
GT & 2.7 & 0.105 & 15.1 & $0.567-402.1$ \\
GG & & & & \\
\hline BMI $\left(\mathrm{kg} / \mathrm{m}^{2}\right)$ & 6.02 & 0.004 & 413.9 & $6.7-492.6$ \\
\hline HBA1C $\%$ & 4.2 & 0.069 & 67.1 & $0.72-256.8$ \\
\hline Total Cholesterol (mg/dl) & 3.98 & 0.003 & 53.6 & $3.8-44.5$ \\
\hline TG(mg/dl) & 1.1 & 0.333 & 3.1 & $0.318-29.5$ \\
\hline LDL-C(mg/dl) & 3.3 & 0.048 & 26.8 & $1.03-95.1$ \\
\hline HDL-C(mg/dl) & -2.04 & 0.297 & 0.13 & $0.003-6$ \\
\hline
\end{tabular}

\section{Discussion}

Obesity is caused by excessive fat accumulation that results from a positive balance between total energy intake and fat catabolism (16). Obesity arises from a complex interaction between genetic variance, environmental, and lifestyle changes. Fat excess is an important predisposing factor for serious medical conditions such as Type 2 Diabetes, cardiovascular disease, stroke, cancer, psychiatric illness, and premature death (17). Several genome-wide association studies (GWAS) have shown the association of common genetic variants with body mass index (BMI). Furthermore, a significant association of some single nucleotide polymorphisms (SNPs) in several genes with obesity has been reported (18). Irisin may contribute to the regulation of energy homeostasis and thus is the potential target for therapy of metabolic dysfunction associated with obesity. This concept is further supported by clinical observations. Circulating irisin was found to be reduced in obese human (19). 
The present study revealed that, there is no significant statistical difference between the two studied groups regarding age and gender. There was a statistically significant difference between the two studied groups regarding serum FBG,HBA1 $1_{C}$, TC,TG ,LDLc, HDLc and BMI . Hosny SS et al ., 2015 who found that highly significant statistical difference in BMI, fasting blood glucose (FBG), HbAlc, total and LDL cholesterol $(\mathrm{p}<0.001)$, and significant statistical difference in triglycerides levels between obese and control groups (20). Obesity was associated with hypertension, hyperglycemia and an atherogenic lipid profile, suggesting that obese individuals have increased susceptibility to metabolic dysfunction and atherosclerosis(21).Hypercholesterolemia was more frequent in obese T2DM subjects. This association was not observed in lean T2DM individuals (22).

Our current study showed that FNDC5 rs3480 (A/G)genotype distribution between the two studied groups showed a significant difference, with increased frequency of the GG,AG and G allele in the patient group and increased AA genotype and A allele frequency in the control group.GG genotype of FNDC5 rs3480 (A/G)increases the risk of obesity by 6.4- fold and AG genotype increases the risk by 3.3 -fold, while the $\mathrm{G}$ allele increases the risk by 2.7 -fold.In FNDC5 rs1746661 (G/T) genotype distribution between the two studied groups showed a significant difference, with increased frequency of the TT and GT genotypes and T allele in the patient group and increased GG genotype and G allele frequency in the control group. As regards TT genotype of FNDC5 rs1746661 (G/T) increases the risk of obesity by 7.9- fold and GT genotype increases the risk by 3.3-fold, while the T allele increases the risk by 4-fold, Park KH et al., (23)has reported that irisin is to be involved in the pathogenesis of several complications of obesity including dyslipidemia, T2DM, arterial hypertension and metabolic Syndrome. However, there are discrepancies regarding the relation of irisin especially with obesity and T2DM. In some studies, a positive association was detected between irisin levels and BMI $(24,25,26)$, while others reported null $(27,28)$.In agreement with Tanisawa et al. (29) showed that the Gallele of rs3480 was associated with increased insulin resistance and its complications. In contrast to our results, Nasser $\mathrm{M}$ et al ., (30) reported that The rs $3480 \mathrm{GG}$ associates with decreased risk of obesity ( $\mathrm{p}=0.005$; odds ratio: 0.48) and lower body mass index (BMI).while rs1746661 variant is associated with elevated triglycerides. Bostrom PA and Sanchis-Gomar F et al., $(31,32)$ reported that irisin may be beneficial not only for the treatment of obesity and T2DM but also for a wide range of pathological conditions characterized by an imbalance between energy intake and expenditure. Furthermore, our study showed that Patients with GG genotype showed higher levels of TC, TG, LDL-c and BMI, while HDL-c was higher in patients with AA genotype. Staiger et al., (33) and Tanisawa et al., (29) did not detect any association between rs3480 and HDLC. However, Tang et al., (34) have reported that rs 3480 has a trend towards association with HDL-C and LDL-C levels in overweight subjects. Moreover, Zhang et al., (35) reported a negative correlation between irisin and intrahepatic triglyceride contents in obese adults. Our study also showed that Patients with TT genotype showed higher levels of TC, TG, LDL-c and BMI, while HDL-c was higher in patients with GG genotype. In agreement with Nasser M et al., (30) reported that rs1746661 variant is associated with elevated triglycerides. The prevalence of hypercholesterolemia was higher in obese T2DM subjects carrying the T allele of rs1746661 than in $\mathrm{G} / \mathrm{G}$ carriers adjusted for age and gender). This association was not observed in lean T2DM subjects (7). Similar results were reported by Ebert T et al., (36) and Panagiotou $\mathrm{G}$ et al., (37) they thought that $\mathrm{T}$ allele of rs1746661 is associated with higher systolic BP, increased TC and LDL-c, and decreased HDL-c in women with T2DM. These results are biologically plausible since different studies have reported associations between plasma or serum irisin levels and lipid profile (TC, LDL-c, HDL-c, and triglycerides levels), FPG levels, and/or BP.

\section{Conclusion}

Our results indicate that GG genotype and G allele of FNDC5 rs3480 (A/G) polymorphism and TT genotype and $\mathrm{T}$ allele of FNDC5 rs1746661 (G/T) might be genetic risk factor for obesity.

\section{References}

[1] Dosaev T, Prakash J, LivshitsG..Contribution of body composition components and soft-tissue biochemical factors to genetic variation of body mass index (BMI) in an ethnically homogeneous population. Am J Hum Biol. 2014; Nov-Dec;26(6):760-767.

[2] Hotta K, Nakata Y, Matsuo T, Kamohara S, Kotani K, Komatsu R, et al. . Variations in the FTO gene are associated with severe obesity in the Japanese. J Hum Genet. 2008;53(6):546-553.

[3] Prakash J, Mittal B, Awasthi S, Agarwal CG, SrivastavaN..Hypoadiponectinemia in obesity: association with insulin resistance. Indian J ClinBiochem. 2013. Apr;28(2):158-163.

[4] Rubenstein AH .Obesity: a modern epidemic. Trans Am ClinClimatolAssoc.2005; 116: 103-111

[5] EckelRH,GrundySM,Zimmet PZ. The metabolic syndrome.Lancet 2005; 365-1415.

[6] T Ebert, S Kralisch, U Wurst, M Scholz, M Stumvoll, P Kovacs, M Fasshauer and ATönjes. Association of metabolic parameters and rs726344 in FNDC5 with serum irisin concentrations.2016; 40: 260-265.

[7] Letícia A. Brondani, Gabriela Boelter, Taís S. Assmann, Cristiane B. Leitão, Luís H. Canani, Daisy Crispim. Irisin-encoding gene (FNDC5) variant is associatedwith changes in blood pressure and lipid profile intype 2 diabetic women but not in men.2015;http://dx.doi.org/10.1016/j.metabol.2015;05.005.

[8] Boström P, Wu J, Jedrychowski MP, Korde A, Ye L, Lo JC et al. A PGC1- $\alpha$-dependent myokine that drives brown-fat-like development of white fat and thermogenesis. Nature 2012; 481: 463-468.

[9] Enyioma O, Michael PT, Abd-IshakurA . Mustapha S and Mona A Leptin, lipid and lipid metabolism related hormones in chronic renal failure in Arabia. Nephrology.2002; 7:115-119. 
[10] Gonen B and RubenstienAH.Determination of glycohemoglobin. Diabetologia.1978;15: 15.

[11] TrinderP.Determination of glucose in blood using glucose oxidase with an alternative oxygen acceptor. J Ann ClinBiochem.1969 ; 6: $24-25$.

[12] Rifai N and WarnickR.Lipids, lipoproteins, apolipoproteins and other cardiovascular risk factors. In: Tietz Textbook of Clinical Chemistry and Molecular Diagnosis. Carl, A. B, Edward, R. A and David, E. B [edrs.]. Saunders. [4th edition]2006 ;Ch 26. PP. 918-922.

[13] Gordon T ,Zidek W and Amer M . Determination of high density lipoprotein cholesterol. J. Med. 1977 ; 42: 707:710.

[14] FriedewaldW, Levy R and Fredrickson D . Estimation of the concentration of low density lipoprotein cholesterol in plasma, without use of the preparative ultracentrifuge.ClinChem.1972;18:499- 502.

[15] Fossati P and Prenciphe L. Determination of serum triglyceride. ClinChem1982; $28: 207-210$.

[16] Sook-Ha Fan and Yee-How Say. Leptin and leptin receptor gene polymorphisms and their association with plasma leptin levels and obesity in a multi-ethnic Malaysian suburban population. 2014; 33(1): 15.

[17] Nguyen DM, El-Serag HB. The epidemiology of obesity.GastroenterolClin North Am. 2010;39:1-7.

[18] Marcela Ulloa-Martínez, Ana I. Burguete-García, SelvasankarMurugesan, Carlos Hoyo-Vadillo, Miguel Cruz-Lopez, Jaime GarcíaMena. Expression of candidate genes associated with obesity in peripheral white blood cells of Mexican children. Arch Med Sci 2016; 12, 5: 968-976.

[19] Hong Tang, Ruili Yu, Shiying Liu, BahetiyaerHuwatibieke, ZiruLi,Weizhen Zhang. Irisin Inhibits Hepatic Cholesterol Synthesis via AMPK-SREBP2 Signaling. EBioMedicine 6.2016; 139-148.

[20] Hosny SS, El-Sherbeny AA, Elewa AA, Kamal El -Dine MM. Retinol Binding Protein-4: a Novel Predictor of Insulin Resistance in Lean Subjects. Int. J. Adv. Res. Biol.Sci. 2(6): 2015; 18-26.

[21] Cornier MA, Dabelea D, Hernandez TL, Lindstrom RC, Steig AJ, Stob NR, et al. The metabolic syndrome.Endocr Rev. 2008;29:777-822.

[22] Letícia A. Brondani, Gabriela Boelter, Taís S. Assmann, Cristiane B. Leitão, Luís H. Canani, Daisy Crispim. Irisin-encoding gene (FNDC5) variant is associated with changes in blood pressure and lipid profile intype 2 diabetic women but not in men. 2015; http://dx.doi.org/10.1016/j.metabol.2015.05.005.

[23] Park KH, Zaichenko L, Brinkoetter M, Thakkar B, Sahin-Efe A, Joung KE, etal.Circulating Irisin in relation to insulin resistance and the metabolic syndrome. J ClinEndocrinolMetab. 2013;98:4899-907.

[24] Huh JY, Panagiotou G, Mougios V, Brinkoetter M, Vamvini MT, Schneider BE, et al. FNDC5 and irisin in humans: I. Predictors of circulating concentrations in serum and plasma and II. mRNA expression and circulating concentrations in response to weight loss and exercise. Metabolism. 2012;61:1725-38.

[25] Stengel A, Hofmann T, Goebel-Stengel M, Elbelt U, Kobelt P, Klapp BF. Circulating levels of irisin in patients with anorexia nervosa and different stages of obesity-Correlation with body mass index.Peptides. 2013;39:125-30.

[26] Pardo M, Crujeiras AB, Amil M, Aguera Z, Jiménez-Murcia S, Baños R et al.Association of irisin with fat mass, resting energy expenditure, and daily activity in conditions of extreme body mass index. Int J Endocrinol. 2014;2014; 857270.

[27] Anastasilakis AD, Polyzos SA, Saridakis ZG, Kynigopoulos G, Skouvaklidou EC, Molyvas D, et al. Circulating irisin in healthy, young individuals: day-nightrhythm, effects of food intake and exercise, and associations with gender,physical activity, diet, and body composition. J ClinEndocrinol Metab.2014;99:3247-55.

[28] Sanchis-Gomar F, Alis R, Pareja-Galeano H, Sola E, Victor VM, Rocha M, et al. Circulating irisin levels are not correlated with BMI, age, and other biological parameters in obese and diabetic patients. Endocrine. 2014;46:674-7.

[29] Tanisawa K, Taniguchi H, Sun X, Ito T, Cao ZB, Sakamoto S, et al. Common single nucleotide polymorphisms in the FNDC5 gene are associated with glucose metabolism but do not affect serum irisin levels in Japanese men with low fitness levels. Metabolism 2014;63:574-83.

[30] Nasser M. Al-Daghri, Abdul Khader Mohammed, Omar S. Al-Attas, Osama E. Amer, Mario Clerici, AmalAlenad and Majed S. Alokail, SNPs in FNDC5 (irisin) are associated with obesity and modulation of glucose and lipid metabolism in Saudi subjects. (2016); 15:54.

[31] Bostrom PA, Fernandez-Real JM, Mantzoros C. Irisin in humans: recent advances and questions for future research.Metabolism 2014;63:178-80.

[32] Sanchis-Gomar F, Lippi G, Mayero S, Perez-Quilis C, García-Giménez JL. Irisin: a new potential hormonal target for the treatment of obesity and type 2 diabetes. J Diabetes 2012;4:196.

[33] Staiger H, Böhm A, Scheler M, Berti L, Machann J, Schick F, et al. Common genetic variation in the human FNDC5 locus, encoding the novel musclederived 'browning'factor irisin, determines insulin sensitivity. PLoS One. 2013;8:e61903.

[34] Tang S, Zhang R, Jiang F, Wang J, Chen M, Peng D, et al. An Interaction between a FNDC5 Variant and Obesity Modulates Glucose Metabolism in a Chinese Han Population. PLoS One. 2014;9:e109957.

[35] Zhang HJ, Zhang XF, Ma ZM, Pan LL, Chen Z, Han HW, et al. Irisin is inversely associated with intrahepatic triglyceride contents in obese adults. J Hepatol 2013;59:557-62.

[36] Ebert T, Focke D, PetroffD, Wurst U, Richter J, Bachmann A, et al. Serumlevels of themyokine irisin in relation tometabolic and renal function. Eur J Endocrinol 2014;170:501-6.

[37] PanagiotouG,MuL, Na B, MukamalKJ, Mantzoros CS. Circulating irisin, omentin-1, and lipoprotein subparticles in adults at higher cardiovascular risk. Metabolism 2014;63:1265-71. 